\title{
山荷叶素异羟弜酸和硫醇衍生物的合成与抑制肿瘤细胞增殖活性
}

\author{
赵育 倪春燕张虞婷 朱 俐* \\ (南通大学航海医学研究所 南通 226001)
}

\begin{abstract}
摘要 通过药效团整合策略将天然抗肿瘤活性木脂素山荷叶素与组蛋白去乙酰化酶抑制剂的药效团长链异羟肜酸或 长链硫醇进行拼合, 得到了 7 个未见文献报道的山荷叶素衍生物, 通过核磁共振氢谱、碳谱、高分辨质谱等方法进行 了结构表征. 并采用了噻唑蓝法(MTT)法对这些化合物的体外抑制肿瘤细胞增殖活性进行了测试, 异羟肟酸类衍生物 具有较强的活性.
\end{abstract}

关键词 山荷叶素; 组蛋白去乙酰化酶抑制剂; 抗肿瘤; 合成

\section{Synthesis and Antiproliferatory Activity of Novel Diphyllin Hydroxamic Acid and Mercaptan Derivatives}

\author{
Zhao, Yu Ni, Chunyan Zhang, Yuting Zhu, Li* \\ (Institute of Nautical Medicine, Nantong University, Nantong 226001)
}

\begin{abstract}
Seven novel diphyllin derivatives have been synthesized by incorporating histone deacetylase inhibitory functional groups into diphyllin. The structures were confirmed by ${ }^{1} \mathrm{H}$ NMR, ${ }^{13} \mathrm{C}$ NMR and HRMS. Their antiproliferative activity against three human cancer cell lines was investigated by methyl thiazolyl tetrazoliym (MTT) assay.

Keywords diphyllin; histone deacetylase inhibitors; antitumor; synthesis
\end{abstract}

组蛋白的可逆乙酰化是调节多种蛋白活性的转录 后修饰的重要过程. 组蛋白去乙酰化酶抑制剂(HDACI) 可以使染色质组蛋白乙酰化水平提高, 导致特定基因表 达激活, 相应地导致细胞的末端分化或癌细胞的调亡, 因此组蛋白去乙酰化酶(HDAC) 已成为抗肿瘤药物研发 领域重要的靶标 ${ }^{[1,2]}$. 目前上市 HDACI SAHA $(N$-差基$N^{\prime}$-苯基辛二酰胺)为广谱 HDACI, 具有明显的胃、血液、 心脏以及神经毒性. 随着人们对 HDAC 认识的不断深 入, 目前研究热点已经转向具有亚型选择性的 HDACI 和与具有多靶点作用的 $\mathrm{HDACI}^{[3 \sim 8]}$.

山荷叶素(diphyllin)是具有 4 位羟基的一种菜基木 脂素, 研究表明山荷叶素的衍生物具有优异的抗肿瘤活 性, 其作用机制与抑制拓扑异构酶 II (Topo II) 和影响微 管组装有关 ${ }^{[9,10]}$. 随着对肿瘤信号转导通路研究的不断 深入, 人们发现通过单一肿瘤靶点的抑制难以达到理想 的阻断肿瘤发生发展通路的效果, 因此作用于多靶点的 抗肿瘤药物引起高度关注. 研究表明同时抑制 HDAC
和 Topo II 具有明显的协同增效作用 ${ }^{[11]}$. 我们采用药效 团整合策略将 diphyllin 和 HDACI 的药效团进行整合, 以 diphyllin 作为 HDACI 的 CAP 区，分别以异羟肜酸和 硫醇 ${ }^{[12]}$ 作为锌离子鳌合基团(ZBG)，设计并合成两类多 靶点 HDACI 抑制剂 1 7, 实现多靶点抗肿瘤的目的(见 图 1).

\section{1 结果与讨论}

\section{1 化学合成}

异羟肜酸类衍生物的合成是将山荷叶素与溴代脂 肪酸乙酯在 $\mathrm{K}_{2} \mathrm{CO}_{3}$ 和相转移催化剂四丁基碘化铵 (TBAI)的作用下成醚 $8 \sim 11$, 然后在 $\mathrm{LiOH}$ 的作用下酯 基水解, 得到酸 $12 \sim 15$, 最后采用 2-(7-偶氮苯并三 唑)- $N, N, N^{\prime}, N^{\prime}$-四甲基脲六氟磷酸酯(HATU)和 $N, N$-二异 丙基乙胺(DIPEA)的缩合体系, 将酸与盐酸着胺缩合得 到异羟肟酸 1 4(图 2). 我们首先采用中间体 $8 \sim 11$ 直

* E-mail: zhulili65@163.com

Received August 31, 2012; revised September 24, 2012; published online October 9, 2012.

Project supported by the Natural Science Foundation of Jiangsu Province (No. BK2011390) and the Priority Academic Program Development of Jiangsu Higher Education Instititutions (PAPD).

江苏省自然科学基金(No. BK2011390)、江苏省高校优势学科(PAPD)资助项目. 

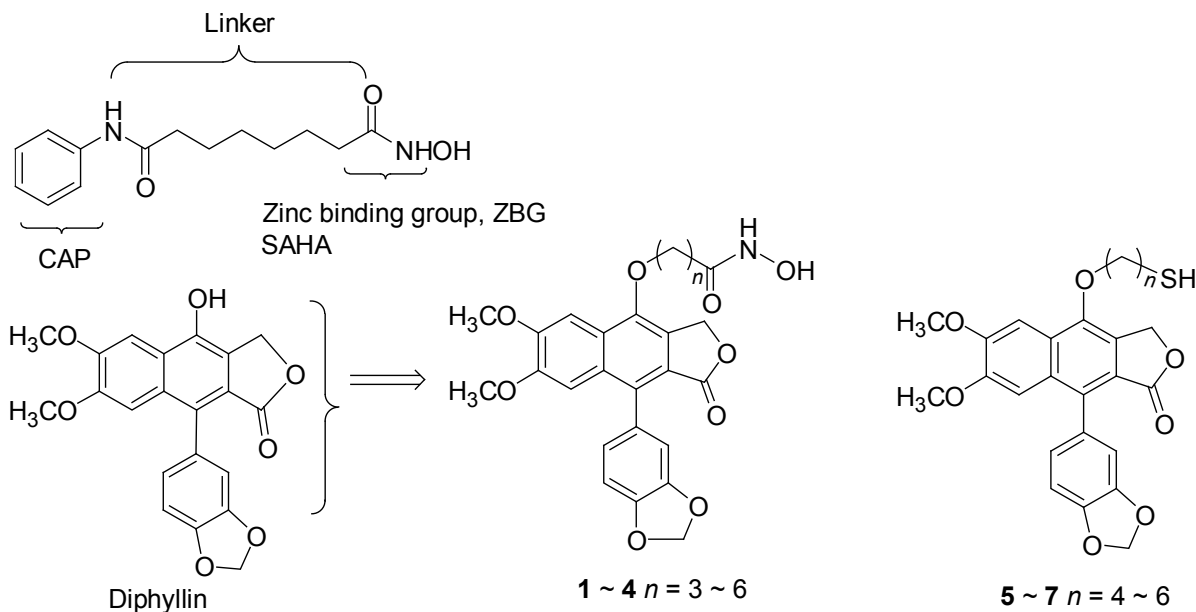

图 1 目标化合物异羟肜酸 1 4 与硫醇 5 7

Figure 1 Hydroxamic acids $\mathbf{1} \sim \mathbf{4}$ and mercaptans $5 \sim 7$

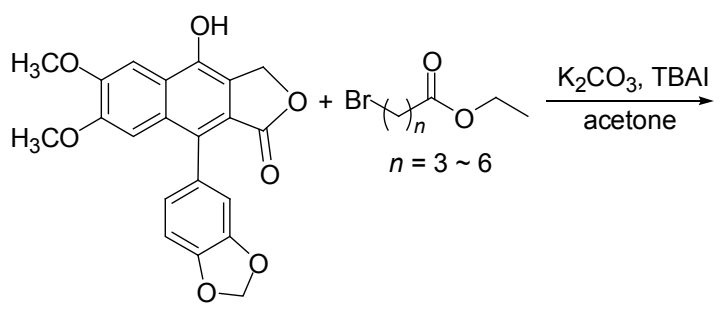<smiles>CCCCCOc1c2cc(OC)c(OC)cc2c(-c2ccc3c(c2)OCO3)c2c(=O)oc(=O)oc12</smiles>

$\begin{aligned} \mathrm{LiOH} \longrightarrow & 8 \sim 11: \mathrm{R}=\mathrm{Et} \\ \longrightarrow & 12 \sim 15: \mathrm{R}=\mathrm{H}\end{aligned}$

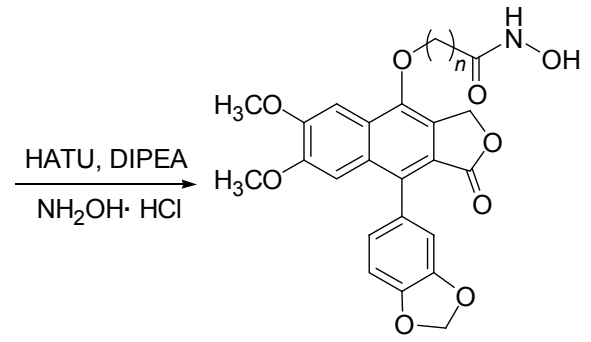

$1 \sim 4 n=3 \sim 6$

图 2 山荷叶素异着弪肜酸衍生物 1 4 的合成

Figure 2 Synthesis of hydroxamic acids diphyllin derivatives $1 \sim 4$

接在甲醇中与现场制备的羟胺进行的氨解反应来制备 目标化合物异羟肜酸, 反应过程中发现由于该中间体在 甲醇中溶解性较差, 氨解反应不能顺利进行. 于是采用 了先酯水解得到酸, 然后在缩合剂的作用下进行缩合, 顺利地制备得到目标化合物 1 4.

硫醇类衍生物的合成是将山荷叶素与二澳烷烃在 $\mathrm{K}_{2} \mathrm{CO}_{3}$ 的作用下生成醚 16 18. 然后与硫代乙酸钾发生 取代反应, 得到硫代乙酸酯 19 21, 最后在碱性条件下 脱除乙酰基, 得到目标化合物 5 7(图 3)在最后一步脱 除乙酰基的反应中发现，山荷叶素的内酯结构在 $\mathrm{NaOH，}$ $\mathrm{NaOMe}$ 等碱的作用下中极易被破坏，导致产率过低， 最后严格控制加入 $\mathrm{NaOH}$ 的量, 使反应体系碱性适中, 完成了乙酰基的脱除.

\section{2 生物活性}

我们以 TopoII 抑制剂 Etoposide 和抗微管药物紫杉 醇(Paclitaxel)作为阳性对照, 选取对山荷叶素类化合物 较为敏感的两种实体瘤细胞, 人结肠癌细胞 HCT116 和 人非小细胞肺癌细胞 $\mathrm{A} 549$, 以及对紫杉醇耐药人非小 细胞肺癌细胞细胞 $\mathrm{A} 549 / \mathrm{T}$ 三种肿瘤细胞进行了 MTT 测
试, 考察 7 个目标化合物抑制肿瘤细胞增殖活性(表 1).

表 1 山荷叶素衍生物 1 7 对三种肿瘤细胞的增殖抑制活性 Table 1 Antiproliferatory activity against three cancer cell lines of diphyllin derivatives $\mathbf{1} \sim \mathbf{7}$

\begin{tabular}{cccc}
\hline \multirow{2}{*}{ Compd. } & \multicolumn{3}{c}{ Cancer cells $\mathrm{IC}_{50} /\left(\mu \mathrm{mol} \cdot \mathrm{L}^{-1}\right)$} \\
\cline { 2 - 4 } & $\mathrm{HCT116}$ & $\mathrm{A} 549$ & $\mathrm{~A} 549 / \mathrm{T}$ \\
\hline 1 & 10.3 & 14.1 & 8.3 \\
2 & 8.1 & 10.4 & 7.8 \\
3 & 1.2 & 6.9 & 7.3 \\
4 & 7.5 & 8.1 & 7.8 \\
5 & $>100$ & $>100$ & $>100$ \\
6 & $>100$ & $>100$ & $>100$ \\
7 & $>100$ & $>100$ & $>100$ \\
Paclitaxel & 1.1 & 0.1 & $>100$ \\
Etoposide & 13.5 & 21.4 & 85.8 \\
\hline
\end{tabular}

MTT 测试结果表明，山荷叶素异羟肜酸衍生物 1 4 对 HCT116, A549 细胞的抑制增殖活性比 Etoposide 更 强, 但是比紫杉醇活性弱. 对于紫杉醇耐药细胞株 A549/T 的抑制增殖活性与 A549 细胞接近, 这表明这些 化合物的抑制增殖机制与紫杉醇不同，对紫杉醇耐药细 胞同样有效. 这 4 个异羟肜酸衍生物对 HCT116 细胞的 

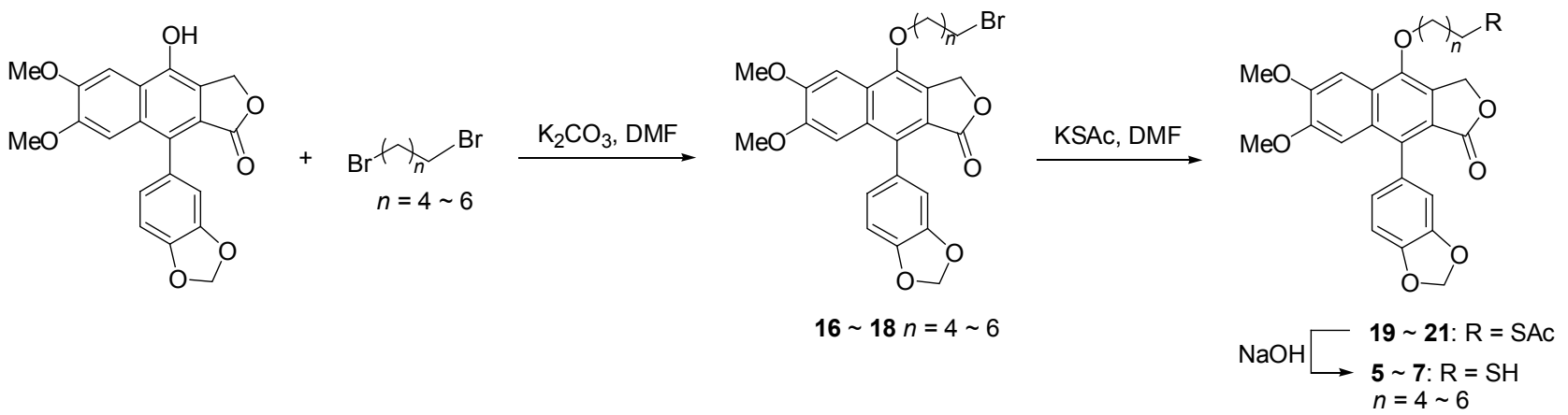

图 3 山荷叶素硫醇衍生物 5 7 的合成

Figure 3 Synthesis of mercaptans diphyllin derivatives $5 \sim 7$

抑制增殖活性要强于 A549 细胞, 其中化合物 3 活性最 好. 表明该类化合物抑制肿瘤细胞增殖的活性与连接臂 的链长有关, 其中己酸衍生物的活性最佳, 丁酸衍生物 活性最弱. 而山荷叶素硫醇衍生物 5 7 对于所测试的 三种肿瘤细胞的抑制增殖活性都比较弱, 说明异羟肜酸 取代基活性优于硫醇取代基. 这 7 个化合物对三种肿瘤 细胞的增殖抑制没有明显的选择性.

\section{2 结论}

我们以山荷叶素为原料, 合成了异羟肜酸和硫醇两 类共 7 个山荷叶素衍生物. 并对这些化合物进行了结构 确证. MTT 实验表明, 山荷叶素异羟肜酸衍生物对所测 试的三种肿瘤细胞都具有一定的抑制增殖活性, 并对紫 杉醇耐药细胞有效. 该类化合物抑制肿瘤细胞增殖的活 性与连接臂的链长有关, 其中己酸衍生物的活性最佳, 丁酸衍生物活性最弱. 所合成的山荷叶素硫醇衍生物未 显示出对所测试的肿瘤细胞的增殖抑制活性. 异羟肜酸 类衍生物有必要进行进一步的药理学研究, 包括对 HDAC 酶的抑制活性以及荷瘤动物模型实验.

\section{3 实验部分}

\section{1 仪器与试剂}

${ }^{1} \mathrm{H}$ NMR 和 ${ }^{13} \mathrm{C}$ NMR 采用 Bruker ARX-300 型核磁 共振仪 (TMS 为内标) 测定; 质谱采用 Q-TOF Global Mass 质谱仪测定. 所用试剂均为市售化学纯或分析纯.

\section{2 化合物合成}

\subsubsection{4-山荷叶素氧基烷酸乙酯 $(\mathbf{8} \sim 11)$ 的合成}

将 $2 \mathrm{mmol}$ diphyllin 溶于丙酮 $60 \mathrm{~mL}$ 中, 依次加入 5 mmol $\mathrm{K}_{2} \mathrm{CO}_{3}, 0.2 \mathrm{mmol}$ TBAI 和 $4 \mathrm{mmol}$ 溴代烷酸乙酯, 在 $\mathrm{N}_{2}$ 保护下于 $56{ }^{\circ} \mathrm{C}$ 反应 $3 \mathrm{~h}$. 反应结束后, 旋蒸除去溶 剂丙酮, 用乙酸乙酯 $30 \mathrm{~mL}$ 稀释残液, 去离子水洗涤 $(50$ $\mathrm{mL} \times 3)$, 无水硫酸钠干燥, 过滤, 浓缩, 柱层析 $[V($ 乙酸 乙酯)： $V$ (石油醚 $)=1: 3$ ]得到固体.
4-山荷叶素氧基丁酸乙酯(8): 产率 $62 \% .{ }^{1} \mathrm{H}$ NMR $\left(\mathrm{CDCl}_{3}, 300 \mathrm{MHz}\right) \delta: 7.52(\mathrm{~s}, 1 \mathrm{H}, 5-\mathrm{ArH}), 7.04(\mathrm{~s}, 1 \mathrm{H}$, 8 -ArH), 6.95 (d, $\left.J=7.8 \mathrm{~Hz}, 1 \mathrm{H}, 2^{\prime}-\mathrm{ArH}\right), 6.80 \sim 6.76(\mathrm{~m}$, $\left.2 \mathrm{H}, 5^{\prime}, 66^{\prime}-\mathrm{ArH}\right), 6.07$ (d, $\left.J=14.4 \mathrm{~Hz}, 2 \mathrm{H}, \mathrm{CH}_{2}\right), 5.45(\mathrm{~s}, 2 \mathrm{H}$, $\left.\mathrm{CH}_{2}\right), 4.27\left(\mathrm{t}, J=6.3 \mathrm{~Hz}, 2 \mathrm{H}, \mathrm{CH}_{2}\right), 4.18(\mathrm{t}, J=7.2 \mathrm{~Hz}, 2 \mathrm{H}$, $\left.\mathrm{CH}_{2}\right), 4.06$ (s, 3H, 6- $\left.-\mathrm{OCH}_{3}\right), 3.78$ (s, 3H, 7- $\left.-\mathrm{OCH}_{3}\right), 2.63$ (t, $\left.J=7.2 \mathrm{~Hz}, 2 \mathrm{H}, \mathrm{CH}_{2}\right), 2.26\left(\mathrm{dd}, J=6.3,12.9 \mathrm{~Hz}, 2 \mathrm{H}, \mathrm{CH}_{2}\right.$ ), 1.24 (t, $J=6.9 \mathrm{~Hz}, 3 \mathrm{H}, \mathrm{CH}_{3}$ ); ESI-MS $m / z: 495[\mathrm{M}+\mathrm{H}]^{+}$.

4-山荷叶素氧基戊酸乙酯(9): 产率 $67 \% .{ }^{1} \mathrm{H}$ NMR $\left(\mathrm{CDCl}_{3}, 300 \mathrm{MHz}\right) \delta: 7.54(\mathrm{~s}, 1 \mathrm{H}, 5-\mathrm{ArH}), 7.04(\mathrm{~s}, 1 \mathrm{H}$, 8 -ArH), 6.95 (d, $\left.J=8.1 \mathrm{~Hz}, 1 \mathrm{H}, 2^{\prime}-\mathrm{ArH}\right), 6.80 \sim 6.79(\mathrm{~m}$, $\left.2 \mathrm{H}, 5^{\prime}, 66^{\prime}-\mathrm{ArH}\right), 6.07$ (d, $\left.J=12.9 \mathrm{~Hz}, 2 \mathrm{H}, 7^{\prime}-\mathrm{CH}_{2}\right), 5.46$ (s, $\left.2 \mathrm{H}, \mathrm{CH}_{2}\right), 4.22\left(\mathrm{t}, J=5.7 \mathrm{~Hz}, 2 \mathrm{H}, \mathrm{CH}_{2}\right), 4.11$ (t, $J=7.5 \mathrm{~Hz}$, $\left.2 \mathrm{H}, \mathrm{CH}_{2}\right), 4.05\left(\mathrm{~s}, 3 \mathrm{H}, 6-\mathrm{OCH}_{3}\right), 3.88(\mathrm{~d}, J=5.1 \mathrm{~Hz}, 2 \mathrm{H}$, $\left.\mathrm{CH}_{2}\right), 3.79$ (s, $\left.3 \mathrm{H}, 7-\mathrm{OCH}_{3}\right), 1.95\left(\mathrm{~s}, 2 \mathrm{H}, \mathrm{CH}_{2}\right), 1.56(\mathrm{~s}, 2 \mathrm{H}$, $\mathrm{CH}_{2}$ ), $1.24\left(\mathrm{t}, J=3.6 \mathrm{~Hz}, 3 \mathrm{H}, \mathrm{CH}_{3}\right.$ ); ESI-MS $m / z: 509$ $[\mathrm{M}+\mathrm{H}]^{+}$.

4-山荷叶素氧基己酸乙酯(10): 产率 64\%. ${ }^{1} \mathrm{H}$ NMR $\left(\mathrm{CDCl}_{3}, 300 \mathrm{MHz}\right) \delta: 7.52(\mathrm{~s}, 1 \mathrm{H}, 5-\mathrm{ArH}), 7.04(\mathrm{~s}, 1 \mathrm{H}$, 8-ArH), 6.95 (d, $\left.J=8.1 \mathrm{~Hz}, 1 \mathrm{H}, 2^{\prime}-\mathrm{ArH}\right), 6.80 \sim 6.76(\mathrm{~m}$, $2 \mathrm{H}, 5$ ',6'-ArH), 6.07 (dd, $J=1.2,14.1 \mathrm{~Hz}, 2 \mathrm{H}, \mathrm{CH}_{2}$ ), 5.46 (s, $2 \mathrm{H}, \mathrm{CH}_{2}$ ), 4.22 (t, $J=6.3 \mathrm{~Hz}, 2 \mathrm{H}, \mathrm{CH}_{2}$ ), 4.12 (q, $J=7.2$ $\left.\mathrm{Hz}, 2 \mathrm{H}, \mathrm{CH}_{2}\right), 4.04$ (s, 3H, 6- $\mathrm{OCH}_{3}$ ), 3.78 (s, 3H, 7- $\mathrm{OCH}_{3}$ ), 2.37 (t, $J=7.2 \mathrm{~Hz}, 2 \mathrm{H}, \mathrm{CH}_{2}$ ), 1.95 (q, $J=6.9 \mathrm{~Hz}, 2 \mathrm{H}$, $\mathrm{CH}_{2}$ ), 1.78 (t, $J=8.7 \mathrm{~Hz}, 2 \mathrm{H}, \mathrm{CH}_{2}$ ), 1.64 (q, $J=6.6 \mathrm{~Hz}$, $\left.2 \mathrm{H}, \mathrm{CH}_{2}\right), 1.25$ (t, $J=3.6 \mathrm{~Hz}, 3 \mathrm{H}, \mathrm{CH}_{3}$ ); ESI-MS $m / z: 523$ $[\mathrm{M}+\mathrm{H}]^{+}$.

4-山荷叶素氧基庚酸乙酯(11): 产率 69\%. ${ }^{1} \mathrm{H}$ NMR $\left(\mathrm{CDCl}_{3}, 300 \mathrm{MHz}\right) \delta: 7.53(\mathrm{~s}, 1 \mathrm{H}, 5-\mathrm{ArH}), 7.04(\mathrm{~s}, 1 \mathrm{H}$, 8-ArH), 6.95 (d, $J=7.5 \mathrm{~Hz}, 1 \mathrm{H}, 2^{\prime}-\mathrm{ArH}$ ), $6.81 \sim 6.79$ (m, 2H, 5',6'-ArH), 6.07 (dd, $\left.J=1.5,14.4 \mathrm{~Hz}, 2 \mathrm{H}, \mathrm{CH}_{2}\right), 5.46$ (s, $\left.2 \mathrm{H}, \mathrm{CH}_{2}\right), 4.20\left(\mathrm{t}, J=6.3 \mathrm{~Hz}, 2 \mathrm{H}, \mathrm{CH}_{2}\right), 4.12(\mathrm{t}, J=7.2$ $\mathrm{Hz}, 2 \mathrm{H}, \mathrm{CH}_{2}$ ), 4.04 (s, 3H, 6- $-\mathrm{OCH}_{3}$ ), 3.79 (s, 3H, 7- $-\mathrm{OCH}_{3}$ ), $2.31\left(\mathrm{t}, J=7.5 \mathrm{~Hz}, 2 \mathrm{H}, \mathrm{CH}_{2}\right), 1.91\left(\mathrm{t}, J=6.6 \mathrm{~Hz}, 2 \mathrm{H}, \mathrm{CH}_{2}\right)$, 
$1.68\left(\mathrm{t}, J=7.2 \mathrm{~Hz}, 2 \mathrm{H}, \mathrm{CH}_{2}\right), 1.61\left(\mathrm{t}, J=5.1 \mathrm{~Hz}, 2 \mathrm{H}, \mathrm{CH}_{2}\right)$, $1.46\left(\mathrm{t}, J=7.2 \mathrm{~Hz}, 2 \mathrm{H}, \mathrm{CH}_{2}\right), 1.24\left(\mathrm{t}, J=5.1 \mathrm{~Hz}, 3 \mathrm{H}, \mathrm{CH}_{3}\right)$; ESI-MS $m / z: 537[\mathrm{M}+\mathrm{H}]^{+}$.

\section{2 .2 山荷叶素异羟肜酸衍生物 1 4 的合成}

将 $1 \mathrm{mmol} 4$-山荷叶素氧基烷酸乙酯溶于 $20 \mathrm{~mL}$ 甲 醇中, 加入 $10 \mathrm{mmol} \mathrm{LiOH}$, 在 $\mathrm{N}_{2}$ 保护下于 $60{ }^{\circ} \mathrm{C}$ 搅拌 3 $\mathrm{h}, \mathrm{TLC}$ 检测至反应结束. 旋蒸除去溶剂甲醇, 用水稀释, $1 \mathrm{~mol} \cdot \mathrm{L}^{-1} \mathrm{HCl}$ 酸化, 用 $\mathrm{CH}_{2} \mathrm{Cl}_{2}(50 \mathrm{~mL} \times 3)$ 萃取, 收集有 机相, 无水硫酸钠干燥, 过滤, 浓缩, 柱层析 $V($ 二氯甲 烷) $: V$ (甲醇 $)=40 ： 1]$ 得 4-山荷叶素氧基烷酸, 直接进 行下步缩合反应.

将 $0.5 \mathrm{mmol}$ 4-山荷叶素氧基烷酸, $0.5 \mathrm{mmol}$ 2-(7-偶 氮苯并三唑)- $N, N, N^{\prime}, N^{\prime}$-四甲基嫝六氟磷酸酯(HATU)和

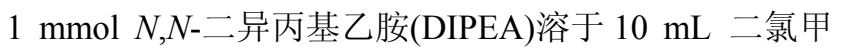
烷(DCM)中, 待溶解后加入 $0.75 \mathrm{mmol}$ 盐酸差胺, 室温 反应 $3 \mathrm{~h}$. 反应结束后, 加入 $10 \mathrm{~mL} \mathrm{CH}_{2} \mathrm{Cl}_{2}$ 稀释, 水洗, 无水硫酸钠干燥, 过滤, 减压浓缩. 将产物用柱层析 $[V($ 二氯甲烷 $): V$ (甲醇 $)=60: 1$ ]纯化得异羟肜酸.

4-山荷叶素氧基丁酸着胺(1): 白色固体, 两步产率 34\%. m.p. 96 99 ${ }^{\circ} \mathrm{C} ;{ }^{1} \mathrm{H}$ NMR $\left(\mathrm{CDCl}_{3}, 300 \mathrm{MHz}\right) \delta$ : 7.36 (s, 1H, 5-ArH), 6.84 (s, 1H, 8-ArH), 6.80 (d, $J=7.2$ $\left.\mathrm{Hz}, 1 \mathrm{H}, 2^{\prime}-\mathrm{ArH}\right), 6.63 \sim 6.56\left(\mathrm{~m}, 2 \mathrm{H}, 5^{\prime}, 6^{\prime}-\mathrm{ArH}\right), 5.90$ (d, $\left.J=10.8 \mathrm{~Hz}, 2 \mathrm{H}, \mathrm{CH}_{2}\right), 5.34$ (s, $\left.2 \mathrm{H}, \mathrm{CH}_{2}\right), 4.12$ (t, $J=6.3$ $\left.\mathrm{Hz}, 2 \mathrm{H}, \mathrm{CH}_{2}\right), 3.95$ (s, 3H, 6- $\left.-\mathrm{OCH}_{3}\right), 3.65$ (s, 3H, 7- $\mathrm{OCH}_{3}$ ), $2.17\left(\mathrm{~s}, 2 \mathrm{H}, \mathrm{CH}_{2}\right), 2.01\left(\mathrm{~s}, 2 \mathrm{H}, \mathrm{CH}_{2}\right) ;{ }^{13} \mathrm{C} \mathrm{NMR}\left(\mathrm{CDCl}_{3}, 75\right.$ MHz) $\delta: 171.3,170.3,151.5,150.1,147.4,146.5,134.3$, $130.3,128.2,126.2,125.4,123.6,118.7,110.7,107.9$, 105.9, 101.3, 100.4, 66.9, 60.5, 56.1, 55.6, 29.7, 25.9; IR (KBr) $v_{\max }: 718,806,926,1167,1224,1259,1381,1457$, 1506, 1562, 1743, 2923, $3459 \mathrm{~cm}^{-1}$; ESI-MS calcd for $\mathrm{C}_{25} \mathrm{H}_{22} \mathrm{NO}_{9}\left(\mathrm{M}^{+}-\mathrm{H}\right)^{-} 480.1372$, found 480.1314 .

4-山荷叶素氧基戊酸羟胺 (2): 白色固体, 两步产率 30\%. m.p. 92 94 ${ }^{\circ} \mathrm{C} ;{ }^{1} \mathrm{H}$ NMR $\left(\mathrm{CDCl}_{3}, 300 \mathrm{MHz}\right) \delta$ : 7.50 (s, 1H, 5-ArH), 7.02 (s, 1H, 8-ArH), 6.94 (d, $J=7.5$ $\left.\mathrm{Hz}, 1 \mathrm{H}, 2^{\prime}-\mathrm{ArH}\right), 6.79 \sim 6.77$ (m, 2H, 5',6'-ArH), 6.07 (d, $J=12.3 \mathrm{~Hz}, 2 \mathrm{H}, \mathrm{CH}_{2}$ ), 5.45 (s, $2 \mathrm{H}, \mathrm{CH}_{2}$ ), 4.22 (t, $J=6.3$ $\left.\mathrm{Hz}, 2 \mathrm{H}, \mathrm{CH}_{2}\right), 4.03$ (s, 3H, 6- $\left.\mathrm{OCH}_{3}\right), 3.77$ (s, 3H, 7- $\mathrm{OCH}_{3}$ ), 1.95 (s, 2H, $\left.\mathrm{CH}_{2}\right), 1.84$ (s, $\left.2 \mathrm{H}, \mathrm{CH}_{2}\right), 1.64\left(\mathrm{~s}, 2 \mathrm{H}, \mathrm{CH}_{2}\right)$; ${ }^{13} \mathrm{C} \mathrm{NMR}\left(\mathrm{CDCl}_{3}, 75 \mathrm{MHz}\right) \delta: 171.2,170.1,151.6,150.2$, $147.5,147.4,146.9,134.4,130.6,128.5,126.4,125.4$, 123.6, 119.1, 110.8, 108.1, 106.2, 101.3, 100.6, 72.3, 66.9, $56.1,55.8,32.5,28.7,25.1$; IR (KBr) $v_{\max }$ : 719, 827, 926, $1168,1229,1258,1453,1505,1556,1748,2927,3452$ $\mathrm{cm}^{-1}$. ESI-MS calcd for $\mathrm{C}_{26} \mathrm{H}_{26} \mathrm{NO}_{9}(\mathrm{M}+\mathrm{H})^{+}$496.1607, found 496.1602 .
4-山荷叶素氧基己酸着胺(3): 白色固体, 两步产率 $35 \%$. m.p. $123 \sim 125{ }^{\circ} \mathrm{C} ;{ }^{1} \mathrm{H}$ NMR $\left(\mathrm{CDCl}_{3}, 300 \mathrm{MHz}\right) \delta$ : 7.45 (s, 1H, 5-ArH), 6.98 (s, 1H, 8-ArH), 6.90 (d, $J=8.1$ $\left.\mathrm{Hz}, 1 \mathrm{H}, 2^{\prime}-\mathrm{ArH}\right), 6.75 \sim 6.70\left(\mathrm{~m}, 2 \mathrm{H}, 5^{\prime}, 6^{\prime}-\mathrm{ArH}\right), 6.04$ (d, $\left.J=11.4 \mathrm{~Hz}, 2 \mathrm{H}, \mathrm{CH}_{2}\right), 5.40\left(\mathrm{~s}, 2 \mathrm{H}, \mathrm{CH}_{2}\right), 4.14$ (t, $J=6.6$ $\mathrm{Hz}, 2 \mathrm{H}, \mathrm{CH}_{2}$ ), 4.00 (s, 3H, 6- $-\mathrm{OCH}_{3}$ ), 3.74 (s, 3H, 7- $\mathrm{OCH}_{3}$ ), 2.17 (s, 2H, $\left.\mathrm{CH}_{2}\right), 1.87$ (s, 2H, $\left.\mathrm{CH}_{2}\right), 1.73\left(\mathrm{~s}, 2 \mathrm{H}, \mathrm{CH}_{2}\right)$, $1.57\left(\mathrm{~s}, 2 \mathrm{H}, \mathrm{CH}_{2}\right) ;{ }^{13} \mathrm{C} \mathrm{NMR}\left(\mathrm{CDCl}_{3}, 75 \mathrm{MHz}\right) \delta: 171.1$, $170.0,151.5,150.2,147.5,147.4,146.9,134.4,130.5$, $128.4,126.4,125.4,123.6,119.0,110.7,108.1,106.1$, 101.3, 100.5, 72.2, 66.9, 56.1, 55.8, 32.6, 29.9, 25.6, 25.1; IR (KBr) $v_{\max }: 710,810,931,1165,1226,1261,1396$, $1457,1505,1562,1649,1758,2923,3446 \mathrm{~cm}^{-1}$. ESI-MS calcd for $\mathrm{C}_{27} \mathrm{H}_{28} \mathrm{NO}_{9}(\mathrm{M}+\mathrm{H})^{+} 510.1764$, found 510.1739 .

4-山荷叶素氧基庚酸着胺(4)：白色固体, 两步产率 $33 \%$. m.p. 93 96 ${ }^{\circ} \mathrm{C} ;{ }^{1} \mathrm{H}$ NMR $\left(\mathrm{CDCl}_{3}, 300 \mathrm{MHz}\right) \delta$ : 7.49 (s, 1H, 5-ArH), 6.99 (s, 1H, 8-ArH), 6.92 (d, $J=7.8$ $\left.\mathrm{Hz}, 1 \mathrm{H}, 2^{\prime}-\mathrm{ArH}\right), 6.77 \sim 6.73\left(\mathrm{~m}, 2 \mathrm{H}, 5^{\prime}, 6^{\prime}-\mathrm{ArH}\right), 6.04(\mathrm{~d}$, $\left.J=12 \mathrm{~Hz}, 2 \mathrm{H}, \mathrm{CH}_{2}\right), 5.43\left(\mathrm{~s}, 2 \mathrm{H}, \mathrm{CH}_{2}\right), 4.18(\mathrm{t}, J=6 \mathrm{~Hz}$, $\left.2 \mathrm{H}, \mathrm{CH}_{2}\right), 4.01$ (s, 3H, 6- $\left.\mathrm{OCH}_{3}\right), 3.76\left(\mathrm{~s}, 3 \mathrm{H}, 7-\mathrm{OCH}_{3}\right)$, $2.15\left(\mathrm{t}, J=6 \mathrm{~Hz}, 2 \mathrm{H}, \mathrm{CH}_{2}\right), 1.89$ (t, $\left.J=6.6 \mathrm{~Hz}, 2 \mathrm{H}, \mathrm{CH}_{2}\right)$, $1.68\left(\mathrm{t}, J=6 \mathrm{~Hz}, 2 \mathrm{H}, \mathrm{CH}_{2}\right), 1.58\left(\mathrm{t}, J=6.3 \mathrm{~Hz}, 2 \mathrm{H}, \mathrm{CH}_{2}\right)$; ${ }^{13} \mathrm{C} \mathrm{NMR}\left(\mathrm{CDCl}_{3}, 75 \mathrm{MHz}\right) \delta: 170.9,169.8,151.5,150.3$, $147.5,147.4,146.9,134.4,130.6,128.5,126.4,125.4$, 123.6, 119.2, 110.8, 108.2, 106.2, 101.2, 100.7, 72.4, 66.8, 56.1, 55.8, 31.9, 29.4, 25.8, 25.8, 25.2; IR (KBr) $v_{\max }: 710$, $810,931,1165,1226,1261,1396,1457,1505,1562,1649$, 1758, 2923, 3446726, 807, 934, 1168, 1226, 1258, 1351, 1450, 1505, 1556, 1649, 1755, 2930, $3429 \mathrm{~cm}^{-1}$. ESI-MS calcd for $\mathrm{C}_{28} \mathrm{H}_{30} \mathrm{NO}_{9}(\mathrm{M}+\mathrm{H})^{+}$524.1920, found 524.1915.

\subsubsection{4-山荷叶素氧基烷基硫醇 $5 \sim 7$ 的合成}

溴代烃中间体 16 18 按照文献[13]方法制备.

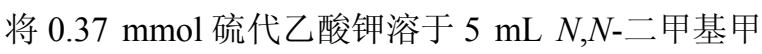
酰胺(DMF)MF 中, 加入 $0.33 \mathrm{mmol}$ 溴代烃中间体 16 18, 室温摚拌 $4 \sim 5 \mathrm{~h}$. 反应结束后, 用乙酸乙酯稀释残 液, 去离子水洗涤 $(50 \mathrm{~mL} \times 3)$, 无水硫酸钠干燥、过滤、 浓缩、柱层析 $[V$ (乙酸乙酯) : $V$ (石油醚 $)=1: 3$ ] 得到 $4-$ 山荷叶素氧基烷基硫代乙酰酯 19 21. 纯化后直接进 行下步脱乙酰基反应.

将 $0.3 \mathrm{mmol}$ 硫代乙酰酯溶于 $10 \mathrm{~mL}$ 甲醇中, 冰浴下 加入 $4 \mathrm{~mL} 0.3 \mathrm{~mol} \cdot \mathrm{L}^{-1} \mathrm{NaOH}$ 溶液, 室温反应 $8 \mathrm{~h}$. 反应 结束后, 加入稀 $\mathrm{HCl}$ 调节 $\mathrm{pH}$ 至 7, 旋蒸除去溶剂甲醇, 用氯仿稀释残液，去离子水洗涤 $(50 \mathrm{~mL} \times 3)$, 无水硫酸 钠干燥, 过滤, 浓缩, 柱层析 $[V$ ( 乙酸乙酯) : $V$ (石油 醚) $=1: 3$ ]得到山荷叶素硫醇衍生物 $5 \sim 7$. 
4-山荷叶素氧基戊基硫醇(5): 白色固体, 两步产率 26\%. m.p. $139 \sim 141{ }^{\circ} \mathrm{C} ;{ }^{1} \mathrm{H}$ NMR $\left(\mathrm{CDCl}_{3}, 300 \mathrm{MHz}\right) \delta$ : 7.52 (s, 1H, 5-ArH), 7.04 (s, 1H, 8-ArH), 6.94 (d, $J=7.8$ $\mathrm{Hz}, 1 \mathrm{H}, 6{ }^{\prime}-\mathrm{ArH}$ ), 6.79 (s, 1H, 2'-ArH), 6.75 (d, $J=7.8 \mathrm{~Hz}$, $\left.1 \mathrm{H}, 5^{\prime}-\mathrm{ArH}\right), 6.06$ (d, J=12.9 Hz, 2H, $\left.\mathrm{CH}_{2}\right), 5.45$ (s, $2 \mathrm{H}$, $\mathrm{CH}_{2}$ ), $4.20\left(\mathrm{t}, J=6.3 \mathrm{~Hz}, 2 \mathrm{H}, \mathrm{CH}_{2}\right), 4.04$ (s, 3H, 6- $\mathrm{OCH}_{3}$ ), $3.78\left(\mathrm{~s}, 3 \mathrm{H}, 7-\mathrm{OCH}_{3}\right), 2.73\left(\mathrm{t}, J=6.3 \mathrm{~Hz}, 2 \mathrm{H}, \mathrm{CH}_{2}\right), 1.95 \sim$ $1.90\left(\mathrm{~m}, 2 \mathrm{H}, \mathrm{CH}_{2}\right), 1.84 \sim 1.79\left(\mathrm{~m}, 2 \mathrm{H}, \mathrm{CH}_{2}\right), 1.75 \sim 1.67$ (m, $\left.2 \mathrm{H}, \mathrm{CH}_{2}\right), 1.58(\mathrm{~s}, 1 \mathrm{H}, \mathrm{SH}) ;{ }^{13} \mathrm{C}$ NMR $\left(\mathrm{CDCl}_{3}, 75\right.$ MHz) $\delta: 169.6,151.6,150.3,147.5,147.4,146.9,134.5$, $130.7,128.5,126.4,125.4,123.6,119.3,110.8,108.2$, 106.2, 101.2, 100.6, 72.3, 66.7, 56.1, 38.7, 29.9, 28.8, 25.1; IR (KBr) $v_{\max }: 726,812,923,1162,1221,1255,1344$, 1452, 1504, 1553, 1648, 1766, 2024, 2922, 3005, 3067 $\mathrm{cm}^{-1}$. MALDI-MS calcd for $\mathrm{C}_{26} \mathrm{H}_{26} \mathrm{O}_{7} \mathrm{SNa}(\mathrm{M}+\mathrm{Na})^{+}$ 505.1297, found 505.1292.

4-山荷叶素氧基己基硫醇(6): 白色固体, 两步产率 36\%. m.p. 101 $103{ }^{\circ} \mathrm{C} ;{ }^{1} \mathrm{H}$ NMR $\left(\mathrm{CDCl}_{3}, 300 \mathrm{MHz}\right) \delta$ : 7.52 (s, 1H, 5-ArH), 7.03 (s, 1H, 8-ArH), 6.94 (d, $J=7.8$ $\left.\mathrm{Hz}, 1 \mathrm{H}, 66^{\prime}-\mathrm{ArH}\right), 6.79$ (s, 1H, 2'-ArH), $6.75(\mathrm{~d}, J=8.1 \mathrm{~Hz}$, $\left.1 \mathrm{H}, 5^{\prime}-\mathrm{ArH}\right), 6.06$ (d, J=13.8 Hz, 2H, $\left.\mathrm{CH}_{2}\right), 5.44(\mathrm{~s}, 2 \mathrm{H}$, $\left.\mathrm{CH}_{2}\right), 4.19$ (t, $\left.J=6.3 \mathrm{~Hz}, 2 \mathrm{H}, \mathrm{CH}_{2}\right), 4.03$ (s, 3H, 6- $\mathrm{OCH}_{3}$ ), $3.78\left(\mathrm{~s}, 3 \mathrm{H}, 7-\mathrm{OCH}_{3}\right), 2.69\left(\mathrm{t}, J=7.2 \mathrm{~Hz}, 2 \mathrm{H}, \mathrm{CH}_{2}\right), 1.95 \sim$ $1.86\left(\mathrm{~m}, 2 \mathrm{H}, \mathrm{CH}_{2}\right), 1.78 \sim 1.69\left(\mathrm{~m}, 2 \mathrm{H}, \mathrm{CH}_{2}\right), 1.63 \sim 1.58$ (m, $\left.2 \mathrm{H}, \mathrm{CH}_{2}\right), 1.56 \sim 1.51\left(\mathrm{~m}, 2 \mathrm{H}, \mathrm{CH}_{2}\right), 1.23(\mathrm{~s}, 1 \mathrm{H}, \mathrm{SH})$; ${ }^{13} \mathrm{C} \mathrm{NMR}\left(\mathrm{CDCl}_{3}, 75 \mathrm{MHz}\right) \delta: 169.7,151.5,150.3,147.5$, 147.4, 147.0, 134.4, 130.6, 128.5, 126.4, 154.4, 123.6, $119.2,110.8,108.2,106.2,101.2,100.6,72.4,66.7,56.0$, $38.7,30.2,29.1,28.2,25.9$; IR (KBr) $v_{\max }: 735,813,932$, 1172, 1224, 1262, 1349, 1459, 1503, 1537, 1618, 1757, 2032, 2926, 3010, $3100 \mathrm{~cm}^{-1}$. MALDI-MS calcd for $\mathrm{C}_{27} \mathrm{H}_{28} \mathrm{O}_{7} \mathrm{SNa}(\mathrm{M}+\mathrm{Na})^{+}$519.1453, found 519.1452.

4-山荷叶素氧基庚基硫醇(7): 白色固体, 两步产率 14\%. m.p. 84 87 ${ }^{\circ} \mathrm{C} ;{ }^{1} \mathrm{H}$ NMR $\left(\mathrm{CDCl}_{3}, 300 \mathrm{MHz}\right) \delta$ : 7.53 (s, 1H, 5-ArH), 7.04 (s, 1H, 8-ArH), 6.94 (d, $J=7.8$ Hz, 1H, 6'-ArH ), 6.79 (s, 1H, 2'-ArH), 6.75 (d, $J=8.1 \mathrm{~Hz}$, $\left.1 \mathrm{H}, 5^{\prime}-\mathrm{ArH}\right), 6.06$ (d, $\left.J=13.8 \mathrm{~Hz}, 2 \mathrm{H}, \mathrm{CH}_{2}\right), 5.45(\mathrm{~s}, 2 \mathrm{H}$, $\left.\mathrm{CH}_{2}\right), 4.21\left(\mathrm{t}, J=6.3 \mathrm{~Hz}, 2 \mathrm{H}, \mathrm{CH}_{2}\right), 4.03\left(\mathrm{~s}, 3 \mathrm{H}, 6-\mathrm{OCH}_{3}\right)$, $3.78\left(\mathrm{~s}, 3 \mathrm{H}, 7-\mathrm{OCH}_{3}\right), 2.69\left(\mathrm{t}, J=6.9 \mathrm{~Hz}, 2 \mathrm{H}, \mathrm{CH}_{2}\right), 1.95 \sim$ $1.87\left(\mathrm{~m}, 2 \mathrm{H}, \mathrm{CH}_{2}\right), 1.75 \sim 1.58\left(\mathrm{~m}, 8 \mathrm{H}, \mathrm{CH}_{2} \times 3\right), 1.43$ (s, $1 \mathrm{H}, \mathrm{SH}) ;{ }^{13} \mathrm{C} \mathrm{NMR}\left(\mathrm{CDCl}_{3}, 75 \mathrm{MHz}\right) \delta: 169.6,151.6$, $150.3,147.5,147.4,146.9,134.5,130.7,128.5,126.4$, 125.4, 119.3, 110.8, 108.2, 106.2, 101.2, 111.6, 72.3, 66.7, 56.1, 38.7, 29.9, 29.7, 28.8, 25.1; IR (KBr) $v_{\max }: 721,811$, 929, 1187, 1225, 1259, 1349, 1459, 1503, 1537, 1618,
1685, 1760, 2854, 2924, 3008, $3091 \mathrm{~cm}^{-1}$; MALDI-MS calcd for $\mathrm{C}_{28} \mathrm{H}_{30} \mathrm{O}_{7} \mathrm{SNa}(\mathrm{M}+\mathrm{Na})^{+}$533.1610, found 533.1592 .

\subsection{MTT 法检测化合物抑制肿瘤细胞增殖活性}

将对数生长期的细胞制成细胞悬液, 用血球计数板 计数并将细胞终浓度稀释至 $1 \sim 2 \times 10^{4} \mathrm{cell} / \mathrm{mL}$, 接种于 96 孔板中, 每孔体积 $100 \mu \mathrm{L}$. 另将不含细胞的孔设为空 白组, 只加入 $100 \mu \mathrm{L}$ 培养基, 孵育 $48 \mathrm{~h}$ 使细胞贴壁. 将 待测的化合物用 DMSO 助溶, 同时用完全培养基稀释 至相应浓度(DMSO 浓度为 $0.1 \%$ ). 肿瘤细胞药物设浓度 梯度浓度, 分别为 $0.01,0.1,1,10,100 \mu \mathrm{mol} \cdot \mathrm{L}^{-1}$, 每孔加 入 $90 \mu \mathrm{L}$ 药液, 每个浓度设 6 个复孔, 药物作用 $72 \mathrm{~h}$. 加 药培养时间结束后, 每孔加入 $10 \mu \mathrm{L}$ MTT 溶液(5 $\mathrm{mg} / \mathrm{mL}), 37{ }^{\circ} \mathrm{C}$ 条件下避光培养 $4 \mathrm{~h}$, 之后每个孔再加入 甲肤溶解液 $100 \mu \mathrm{L}, 37{ }^{\circ} \mathrm{C}$ 下睬育 $5 \sim 6 \mathrm{~h}$, 酶标仪在 570 $\mathrm{nm}$ 波长下检测 $\mathrm{OD}$ 值, 并且计算抑制率. 计算公式如 下:

$$
\begin{aligned}
\text { 抑制率 }= & \text { (对照组 } O D-\text { 处理组 } O D) /(\text { 对照组 } O D- \\
& \text { 空白组 } O D) \times 100 \%
\end{aligned}
$$

采用 Sigma plot 计算 $\mathrm{IC}_{50}$ 值.

\section{References}

[1] Bolden J. E.; Peart, M. J.; Johnstone, R. W. Nat. Rev. Drug Discovery 2006, 5, 769.

[2] Zhang, L.; Fang, H.; Xu, W. F. Med. Res. Rev. 2010, 30, 585.

[3] Qian, C. G.; Lai, C. J.; Bao, R.; Wang, D. G.; Wang, J.; Xu, G. X.; Atoyan, R.; Qu, H.; Yin, L.; Samson, M.; Zifcak, B.; Ma, A. W. S.; DellaRocca, S.; Borek, M.; Zhai, H. X.; Cai, X.; Voi M. Clin Cancer Res. 2010, 18, 4104.

[4] Witt, O.; Deubzer, H. E.; Milde, T.; Oehme, I. Cancer Lett. 2009, 277,8 .

[5] Cai, X.; Zhai, H. X.; Wang, J.; Forrester, J.; Qu, H.; Yin, L.; Lai, C. J.; Bao, R.; Qian, C. G. J. Med. Chem. 2010, 53, 2000.

[6] Chen, L. Q.; Petrelli, R.; Gao, G. Y.; Wilson, D. J.; Mclean, G. T.; Jayaram, H. N.; Sham, Y. Y.; Pankiewicz, K. W. Bioorg. Med. Chem. 2010, 18, 5950.

[7] Passarella, D.; Comi, D.; Vanossi, A.; Paganini, G.; Colombo, F.; Ferrante, L.; Zuco, V.; Danieli, B.; Zunino, F. Bioorg. Med. Chem. Lett. 2009, 19, 6358.

[8] Li, X. H.; Huang, D. W.; Sun, L.; Xiu, Z. L.; Nishino, N. Chin. J. Org. Chem. 2011, 31, 312 (in Chinese).

(李晓晖, 黄大卫, 孙蕾, 修志龙, 西野宪和, 有机化学, 2011, 31, 312.)

[9] Zhao, Y.; Ni, C. Y.; Zhang, Y. T.; Zhu, L. Arch. Pharm. Chem. Life Sci. 2012, 8, 622.

[10] Zhao, Y.; Hui, J.; Wang, D.; Zhu, L. Chem. Pharm. Bull. 2010, 58, 1324.

[11] Guerrant, W.; Patil, V.; Canzoneri, J. C.; Oyelere A. K. J. Med. Chem. 2012, 55, 1465.

[12] Liu, X. H.; Song, H. Y.; Zhang, J. X.; Han, B. C.; Wei, X. N.; Ma, X. H.; Cui, W. K.; Chen, Y. Z. Mol. Inf. 2010, 29, 407.

[13] Zhao, Y.; Wang, D.; Hui, J.; Zhu, L. Med. Chem. Res. 2010, 19, 71.

(Li, L.; Fan, Y.) 